\title{
Novel Phenomena in Charged Bose Liquid
}

\author{
Kimyeong Lee and Oleg Tchernyshyov \\ Department of Physics, Columbia University, New York, NY 10027, USA
}

(November 5, 2018)

\begin{abstract}
We investigate charged Bose liquid immersed in uniform background charge at zero temperature. Novel phenomena, such as oscillatory shielding of external localized electric charge, rotons and charge density waves (charge stripes in two dimensions), occur in any dimensions. Oscillatory shielding is caused by mixing between scalar boson exchange and Coulomb interactions, which mediate opposite forces. On the other hand, rotons and charge density waves are due to attractive local self-interaction of bosons. Rotons can be regarded as a finite size charge density wave packet without any back flow. We also comment on charge stripes observed recently in cuprates and nickelates.
\end{abstract}

74.20.Mn,71.35.Lk,11.15.Ex

Recently there have been several experimental observations of charge and spin stripes in high temperature superconductor materials [1 - 3]. There have been several theoretical discussions about the origin and implications of these stripes [4]. Experiments show on-set of charge order before spin order, showing that charge stripe is primary and spin stripe is secondary. The superconducting transition temperature is either zero in compounds with a static wave of charge density, or significantly reduced when dynamical correlations are observed. It would be interesting to find a simple model which produces charge stripes and dynamical correlations.

In this paper we investigate charged Bose liquid immersed in uniform external electric charge at zero temperature. We describe charged Bose liquid by a complex scalar field with nonlinear local self-coupling and gauge coupling with photons. By analyzing linear fluctuations around homogeneous ground state, we show that novel collective modes, like oscillatory shielding of external localized charges, rotons and charge density waves, occur in any dimensions. We identify physical reason behind these phenomena. Our model can be regarded as a simple toy model which provides such rich phenomena, and may also shed some light on current experiments.

Since unique is the rest frame of uniform external charge, there is no Lorentz or Galilean symmetry in our theory. There have been many works on this system with attention on vortex dynamics [5.67. Vortex dynamics is particularly simplified in the self-dual model [5], where magnetic flux vortices are shown not to carry any intrinsic angular momentum.

The presence of uniform background charge leads to nontrivial consequences. The average charge density of bosons is determined by the condition of electric charge neutrality rather than by the chemical and potential energy. For this reason, the (short-range) self-interaction of bosons does not have to be repulsive for the system to be stable. If this interaction is weakly attractive, low-energy excitations from a uniform ground state are fluctuations of charge density with a characteristic wavelength. These excitations can be called rotons in analogy with those in superfluid helium. As the strength of attraction increases, the frequency of this mode vanishes and the uniform ground state becomes unstable, form- ing charge density waves. In superfluid helium nonlocal attractive self-interaction of helium atom plays a crucial role in the existence of rotons. (For a review of current understanding of rotons in superfluid helium, see Ref. [7].)

A closely related question is the screening of a probe charge. It is normally expected that a localized external charge is fully shielded by charged bosons; the amount of total charge inside a sphere with the external charge in its center falls off exponentially with the radius. However, in the case we study, the shielding can be oscillatory. This originates from mixing of two opposite forces mediated by scalar boson exchange and Coulomb interactions.

We find a link between oscillatory shielding and the existence of a roton dip in the excitation spectrum. As stressed by Feynman [8], the roton minimum is associated with a short-range crystal-like structure in liquid helium. In that case, the density-density correlation $\langle\rho(\mathbf{r}) \rho(0)\rangle$ is a non-monotonic function of the distance, an effect similar to the oscillatory screening of charge.

Within the scope of our model, a roton wave packet can be visualized as a localized charge density wave. As discussed above, it originates from the attractive selfinteraction of bosons. While the wave packet itself is at rest (zero group velocity), charge density waves are moving at the roton phase velocity. Curiously, there is no net flow of charge associated with this motion and therefore it is unnecessary to attach a vortex ring to this wave packet in order to produce a back flow. The idea of back flow goes back to Feynman and Cohen [9], who tried to modify Feynman's original ansatz to enforce the conservation of current. In our theory the charge current is exactly conserved from the start and so there is no need for back flow. At least our result is consistent with the current view of rotons in superfluid helium [7].

We describe charged Bose liquid by a bosonic scalar field $\psi$ with a local self-interaction and the electromagnetic coupling. This liquid is immersed in a uniform background charge $\rho_{e}>0$. The Lagrangian for charged Bose liquid is

$$
\begin{aligned}
\mathcal{L}= & -\frac{1}{16 \pi} F_{\mu \nu} F^{\mu \nu}+i \psi^{*}\left(\partial_{0}-i q A_{0}\right) \psi \\
& -\frac{1}{2 m}\left|\left(\partial_{i}-i q A_{i}\right) \psi\right|^{2}-U\left(|\psi|^{2}\right)-q \rho_{e} A_{0}
\end{aligned}
$$


where $\hbar=c=1$. The Gauss law constraint is

$$
\partial_{i} F_{0 i}+4 \pi q\left(\rho-\rho_{e}\right)=0 .
$$

As the charged background is static, homogeneous and uniform, there are conserved energy and linear and angular momenta. The Noether angular momentum density is not gauge invariant and needs a modification, which plays a crucial role in vortex dynamics [5].

The homogeneous configuration satisfying the field equations is given as

$$
\left\langle A_{\mu}\right\rangle=0 \text { and }\langle\psi\rangle=\sqrt{\rho_{e}} e^{-i \alpha t},
$$

where $\alpha=d U /\left.d \rho\right|_{\rho_{e}}$. Total charge density is equal to zero and the system is charge neutral. We approach this problem by the semiclassical approximation around this uniform background. To find out elementary excitations on this homogeneous configuration, we study linear fluctuation equations in the unitary gauge $\operatorname{Arg} \psi=0$, and so $\left\langle A_{0}\right\rangle=\alpha / q$. In the Fourier mode $\delta \psi, \delta A_{\mu} \sim e^{-i \omega t+i p^{i} x^{i}}$, the linearized field equations become

$$
\begin{aligned}
& -\left(\frac{p^{2}}{2 m}+2 \lambda \rho_{e}\right) \delta \psi+q \sqrt{\rho_{e}} \delta A_{0}=0, \\
& p^{2} \delta A_{0}+\omega p^{i} \delta A_{i}+8 \pi q \sqrt{\rho_{e}} \delta \psi=0, \\
& \left(\omega^{2}-p^{2}-m_{A}^{2}\right) \delta A_{i}+p^{i} p^{j} \delta A_{j}+\omega p^{i} \delta A_{0}=0,
\end{aligned}
$$

where $\lambda=d^{2} U /\left.d \rho^{2}\right|_{\rho_{e}}$ and $m_{A}^{2}=4 \pi \rho_{e} q^{2} / m$. The parameter $\lambda$ is the strength of the local self-coupling for linear fluctuations.

From these equations, we get the dispersion relation for the transverse modes,

$$
\left(\omega^{2}-p^{2}-m_{A}^{2}\right) \epsilon_{i j k} p^{j} A^{k}=0 .
$$

This Anderson-Higgs mechanism explains the Meissner effect with the London penetration length $\lambda_{L}=1 / m_{A}$. The dispersion relation for the mixed mode of $\delta \psi, \delta A_{0}$ and $p^{i} \delta A_{i}$ is

$$
\omega^{2}=m_{A}^{2}+\frac{p^{2}}{2 m}\left(\frac{p^{2}}{2 m}+2 \lambda \rho_{e}\right) .
$$

For the time-independent fluctuations, we get the static relation

$$
p^{2}=-2 m \lambda \rho_{e} \pm 2 m \sqrt{\left(\lambda \rho_{e}\right)^{2}-m_{A}^{2}} .
$$

This describes how the system responds to any timeindependent external source.

Depending on the range where the parameters of the theory lies, we will see that several collective modes are allowed by Eqs. (7) and (8), which are summerized as follows:

$$
\begin{aligned}
\lambda \rho_{e}>m_{A} ; & \text { exponential shielding, } \\
\left|\lambda \rho_{e}\right|<m_{A} ; & \text { oscillatory shielding, } \\
0>\lambda \rho_{e}>-m_{A} ; & \text { rotons, } \\
\lambda \rho_{e}<-m_{A} ; & \text { charge density waves, } \\
\lambda \rho_{e}<<-m_{A} ; & \text { Wigner crystal. }
\end{aligned}
$$

When the electromagnetic coupling constant $e^{2}$ vanishes, this theory has a smooth limit. It describes a neutral Bose liquid with local self-interaction. We know well that with repulsive self-interaction $\lambda>0$, the homogeneous configuration is stable with positive pressure. Elementary excitations in that case are massless phonons with sound speed $v_{s}^{2}=\lambda \rho_{e} / m$. If we have introduced a proper nonlocal self-interaction as in superfluid helium, the roton mode would have appeared. There is no massive mode but there exists a natural length scale, i.e., the coherence length $\xi=1 / \sqrt{4 m \lambda \rho_{e}}$. With attractive self-interaction $\lambda<0$, the configuration is unstable. Bosons aggregate and form droplets, or Q-balls, with mean charge density $\rho_{c}$, at which $U(\rho)$ takes the minimum value.

Let us first study how the system responds to the static localized probe charge. Far away from this source, the field configuration deviates little bit from the uniform configuration (41). Since the charge couples to $A_{0}$, Eq. (8) would govern the behavior of such a deviation. There are three interesting ranges of the parameters from Eq. (8). When the coupling is strongly repulsive so that the parameters lies in the range (9), the solution of Eq. (8) is pure imaginary. Thus the local source is shielded exponentially. However, the solution of Eq. (8) becomes complex when the parameter lies in the range (10). Thus the fields would fall off exponentially but the sign of the field would oscillate, like the amplitude of underdamped harmonic oscillators. We will call this as oscillatory shielding.

To find the physical origin of such oscillatory screening, let us recall the old lore. For two like sources in large separation, the virtual scalar boson exchange leads to the attractive Yukawa force, but the virtual photon exchange leads to the repulsive Coulomb force. For the opposite charges, the scalar force is repulsive and the Coulomb force is attractive. This can be seen directly by obtaining the interacting potentials from the corresponding Lagrangians,

$$
\begin{aligned}
& \mathcal{L}_{1}=-\frac{1}{2}\left(\partial_{i} f\right)^{2}-\frac{1}{2} m_{1}^{2} f^{2}+\rho(x) f, \\
& \mathcal{L}_{2}=\frac{1}{2}\left(\partial_{i} A_{0}\right)^{2}+\frac{1}{2} m_{2}^{2} A_{0}^{2}+\rho(x) A_{0},
\end{aligned}
$$

where $\rho(x)=q_{1} \delta^{3}\left(x-x_{1}\right)+q_{2} \delta\left(x-x_{2}\right)$. Here we have neglected the time dependence and scaled the field variables. The sign of the field propagator is also associated with the sign of the exchange force. Thus the $A_{i}$ exchange would lead to the similar force as the scalar boson exchange. We already know this is true as two parallel currents are attractive and two opposite currents are repulsive. (The magnetic flux of vortices is not a direct source of $A_{i}$ unlike the current. The force between fluxes by the $A_{i}$ exchange is more like the Coulomb case.) When there is a mixing between $A_{0}$ and $f$ by a Lagrangian $\mathcal{L}_{3}=\mu^{2} f A_{0}$, the corresponding static relation of the total Lagrangian $\mathcal{L}_{1}+\mathcal{L}_{2}+\mathcal{L}_{3}$ becomes

$$
\left(p^{2}+m_{1}^{2}\right)\left(p^{2}+m_{2}^{2}\right)+\mu^{4}=0 .
$$


When $2 \mu^{2}>\left|m_{1}^{2}-m_{2}^{2}\right|$ so that the mixing is strong enough, the solution $p^{2}$ changes from negative real numbers to complex number. This is how oscillatory shielding appears.

More interestingly, when the parameters lie in the range (12), the solution of Eq. (8) becomes real numbers. The fluctuations would not die out. Especially near $\lambda \rho_{e} \approx-m_{A}$, the charge density would be oscillating with a characteristic wave length

$$
l_{C D W}=\frac{2 \pi}{\sqrt{2 m m_{A}}} .
$$

This describes charge density waves. (In two dimensions, it would be charge stripes.) In our case $m_{1}^{2}$ and $m_{2}^{2}$ of Eq. (16) can be negative and so the charge density wave mode is possible.

Let us now consider the time-dependent fluctuations described by Eq. (7). The slope of $\omega(p)$ at $p=0$ becomes negative when the self-interaction is attractive so that the parameters lie in the range (11). It has the minimum at

$$
p_{*}=\sqrt{2 m\left(-\lambda \rho_{e}\right)},
$$

with the value

$$
\omega_{*}=\sqrt{m_{A}^{2}-\lambda^{2} \rho_{e}^{2}} .
$$

As long as $\lambda \rho_{e}>-m_{A}$, the mode at the minimum has the positive energy and is called rotons.

For a roton mode, we have to choose a particular direction, $\mathbf{p}_{*}$. We can form the roton wave packet by superposing the plane waves with an envelop function $\mathcal{F}$. Since the dispersion relation distinguishes the parallel and orthogonal momenta with respect to $\mathbf{p}_{*}, \mathcal{F}$ can depend on parallel and orthogonal momenta independently. Especially if $\mathcal{F}$ is peaked at $\mathbf{p}_{*}$, the group velocity would be zero.

Now one may wonder what happens to charge density and current in the roton wave packet, especially whether there exists any back flow. We first note that our theory works in any spatial dimension. Thus we first consider the system in one dimensional space. When the wave packet size is large, the major contribution to charge density and current comes from the $\mathbf{p}_{*}$ plane wave. Inside the wave packet, both positive and negative charge regions of wave length $\lambda_{*}=2 \pi / p_{*}$ moves forward with phase velocity $v_{*}=\omega_{*} / p_{*}$. Thus, there is no net charge transfer from one end to another end of the wave packet, and no need for back flow of charge. This is consistent with the fact that the current conservation holds for linear fluctuations. The charge density wave inside the roton wave packet is exactly like sound wave in fluid, which does not transport any mass density and needs no back flow.

Coming back to the two or three dimensional problem, we note that the envelop function can be chosen to be Gaussian along the parallel and orthogonal directions, independently. Then the above one-dimensional argument shows that there is no back flow even in these cases. If there is any back flow, it may manifest itself as vortex ring, or smoke ring around the roton wave packet, which is clearly nonperturbative and contradicts the perturbative nature of our fluctuations. Smoke ring is also impossible in one-dimensional space.

If the interaction between bosons become more attractive so that Eq. (12) holds, the roton mode becomes unstable and charge density waves appear. The homogeneous field configuration would be no longer the ground state configuration. Since the charge density wave mode is characterized by a single length scale $l_{C D W}$, the ground state is made of a static plane wave of the given wave length, pointing a particular direction, say $\hat{\mathbf{z}}$. This lowers the gradient energy. Thus the translation symmetry is broken along the $\mathrm{z}$ axis but is invariant along the $\mathrm{x}-\mathrm{y}$ plane. The rotational symmetry is also partially broken to the $\mathrm{z}$ directional rotation. There would be a Goldstone boson mode which modulate the position of the crest of charge density waves. The amplitude of charge density waves would be fixed by the nonlinear interaction. The exact semiclassical description of the ground state needs the evaluation of the full nonlinear field equations, which will tell us whether the background charge is exposed at the bottom of the wave. (This may tell us whether this phase is superconducting or metallic along the z-direction).

When the attractive interaction becomes still stronger, the unstable modes can have a range of momentum. We have to treat the full nonlinear field equation to find the exact ground state field configuration. However, one can guess the ground state configuration in this case by starting from the other end where the electromagnetic coupling is negligible. If we turn off the electromagnetic coupling, charged bosons will aggregate to many droplets, or Q-balls, and then they will grow. When we turn on the electromagnetic coupling, they cannot grow forever. Rather Q-balls of suitable size would arrange themselves into a crystal structure to lower the Coulomb energy. This configuration is analogous to the Wigner crystal of electrons in a uniform positive charged background. In this case the translation and rotational symmetries are broken to a discrete crystal group.

The recently discovered charge stripes and dynamical correlations can be regarded as charge density waves and rotons in our context. While their dynamical origin may be far complicated, charged Bose liquid considered here may capture some physics (aside from spin and lattice effects). As the dopping increases in these materials, the carrier charge density changes. There are several possibilities of behavior. Bosons can be repulsive or attractive in small density, though they are expected to be repulsive in high density.

At very low density $m_{A} \sim \sqrt{\rho_{e}} \gg\left|\lambda \rho_{e}\right|$ and therefore the charge shielding is oscillatory with the wave vector $p \approx(i \pm 1) \sqrt{m m_{A}}$. Experimentally it may be difficult to tell this type of shielding from purely exponential because the imaginary part of $p$ equals the real part. If $\lambda$ is positive and approximately constant, the exponential 
screening appears in high density.

Second possibility is that $\lambda$ is negative in small density and so the roton mode presents in low density. As the density $\rho_{e}$ increases, the roton dip becomes more prominent. Being lowest energy excitations of the system, rotons deplete the condensate density at temperatures equal to or above their energy, like dynamical correlations in real material. In higher density the repulsive interaction turns on and the roton mode disappears.

If the attractive interaction remains to high enough densities, the roton mode becomes unstable at some critical density and the system may not be superfluid any longer. At a higher density, the reduced attraction can no longer maintain a static charge density wave and superfluidity comes back. At the moment of instability the wavelength of charge density waves is determined by the boson mass $m$ and the photon mass $m_{A}$ (the London penetration depth). If we take $m$ equal to two electron masses and the London length $\lambda_{L}=2000 \AA$, a typical experimental value, the resulting CDW wavelength (17) is of order

$$
l_{C D W}=\pi \sqrt{\frac{\lambda_{L} \hbar}{m_{e} c}}=8.5 \AA .
$$

This is of the same order as the experimental value $\sim$ $15 \AA$, which is encouraging. (Note that this analysis is bases on linear fluctuations and so the Meissner effect is still true. But this may not be true once the exact semiclassical ground state is found.)

It is surprising that our simple system shows many fascinating phenomena, as oscillatory shielding, rotons and charge density waves. Our work suggests that these phenomena are quite generic. We now have tools to explore quantum mechanics of these collective modes. We can, for example, calculate spin of rotons in our model. It would be also interesting to analyze the system by using the method of many body physics. It is known that when $\lambda=0$ the homogeneous configuration changes to the Wigner crystal in high enough density due to the Coulomb repulsion [10], which we do not see in our approach.

Even though we have not considered here, there exist also magnetic flux vortices in the theory. The naive division between type I and type II should be redrawn by studying the long-distance interaction between magnetic flux vortices in the exponential shielding region.

It would be most interesting to find out whether our consideration has any direct bearing on real superconductors, whose physics was investigated in context of charged Bose liquid [11]. Recently there has been some proposal of more realistic field theoretic models of the BCS superconductors at zero temperature 12]. It would be interesting to find out whether our consideration applies to that case. There are also numerous works relating superconductivity and charge density wave in one-dimensional systems [13]. We hope that our work can provide some useful insights here as well.
Acknowledgment: The work of K.L. was supported in part by the NSF Presidential Young Investigator Fellowship. The work of O.T. was supported in part by the US Department of Energy and Japan NEDO. The part of this work was done in Aspen Center for Physics. We thank Elihu Abrahams, Sidney Coleman, David Ceperley, Miklos Gyulassy, T.D. Lee, John Negele, Michael Stone, and Tomo Uemura for useful discussions.

[1] C.H. Chen, S.-W. Cheong and A.S. Cooper, Phys. Rev. Lett. 71, 2461 (1993); J.M. Tranquada et al., Phys. Rev. B 52, 3581 (1995); V. Sachan et al., Phys. Rev. B 51, 12742 (1995).

[2] J.M. Tranquada et al., Nature 375, 561 (1995); Phys. Rev. B 54, 7489 (1996); J.M. Tranquada et al., Phys. Rev. Lett. 78, 338 (1997).

[3] S-W Cheong et al., Phys. Rev. Lett. 67, 1791 (1991); T.E. Mason, G. Aeppl and H.A. Mook, Phys. Rev. Lett. 68, 1414 (1992); T.R. Thruston et al., Phys. Rev. B 46, 9128 (1992).

[4] For some of recent theoretical discussions, see O. Zachar, S.A. Kivelson and V.J. Emery, Landau Theory of Stripe Phases in Cuprates and Nickelates, cond-mat/9702055; C. Nayak and F. Wilczek, Int. J. Mod. Phys. B10, 2125 (1996).

[5] K. Lee, Phys. Rev. D49, 4265 (1994).

[6] G.N. Stratopoulos and T.N. Tomaras, Physica D89, 136 (1995); M. Stone, Magnus and other forces on vortices in superfluids and superconductors, to appear (1997).

[7] R.J. Donnelly, Phys. World 10 no. 2, 25 (1997).

[8] R.F. Feynman, Phys. Rev. 91, 1301 (1953); Phys. Rev. 94, 262 (1994).

[9] R.F. Feynman and M. Cohen, Phys. Rev. 102, 1189 (1956).

[10] D.M. Ceperley and B.J. Alder, Phys. Rev. Lett. 45, 566 (1980).

[11] A.S. Alexandrov and N.F. Mott, Rep. Prog. Phys. 57, 1197 (1994).

[12] M. Stone, Int. Jour. Mod. Phys. B9, 1359 (1995); I. R. Aitchison, P. Ao, D. J. Thouless and X.-M. Zhu, Phys. Rev. B51, 6531 (1995).

[13] G. Gruner, Density waves in solids, (North-Holland, New York, 1994); S. Kagoshima, H. Nagasawa, T. Sambongi, One-dimensional conductors (Springer-Verlag, Berlin, 1988). 